\title{
HIGH VOLTAGE SMART POWER MODULE FOR FAULT-TOLERANT LAUNCHER APPLICATIONS
}

\author{
Debrouwere Richard $^{(1)}$, Guillaume Michel $^{(1)}$ \\ (1) Thales Alenia Space Belgium, Rue Chapelle Beaussart 101, B-6032 Mont-sur-Marchienne, Email : \\ firstname.name@thalesaleniaspace.com
}

\begin{abstract}
Generalization of electromechanical actuators use in thrust vector control launchers applications has increased electronics power level needs by a factor of ten, in a global context which puts costs under pressure.

The need for a modular and affordable power solution being versatile to fulfil different launcher mission profiles has become thereby stronger.

In order to target different power and functional needs, an attractive design should be modular and multipurpose, will work at higher voltages and will be able to deliver powers up to a dozen of kilowatts.

Moreover, an efficient design to cost approach will consider alternative technologies, widely used by other high-reliability industries, including Components Off-The-Shelf (COTS) integration.

This paper presents the design of a low cost and highly integrated smart power module (SPM) intended to be used into launchers applications, embedding technologies and components from automotive world and being mainly producible by large-scale industry.
\end{abstract}

\section{SPM SPECIFICATION}

\subsection{Introduction}

The presented Smart Power Module (SPM) targets to drive motors but architecture and technologies are intended to be a future base element of a wider range of applications (DC/DC, DC/AC, satellite,...), its versatility being within others provided by embedded intelligence.

The design must be compliant to specific launcher constraints: radiative environment (mainly robustness to SEE, cumulated radiations being negligible), missions profiles (from several minutes into launchers lower stages and up to 30 hours into upper stages), thermal interfaces (from adiabatic conditions during several minutes to conductive interfaces during up to 30 hours) and launchers applications specific fault-tolerance requirements.

\subsection{Functional architecture}

A full $\mathrm{H}$ power bridge has been integrated together with power electronics drive and isolation.

The choice of a $\mathrm{H}$ bridge has been made as it delivers a voltage level that is twice the high power supply one, delivering there through a higher power to the load. Moreover, $\mathrm{H}$ topology will be to manage star shaped

An accurate current measure, several protections and embedded intelligence are associated to power electronics in order to satisfy to applications where control can be highly distributed :

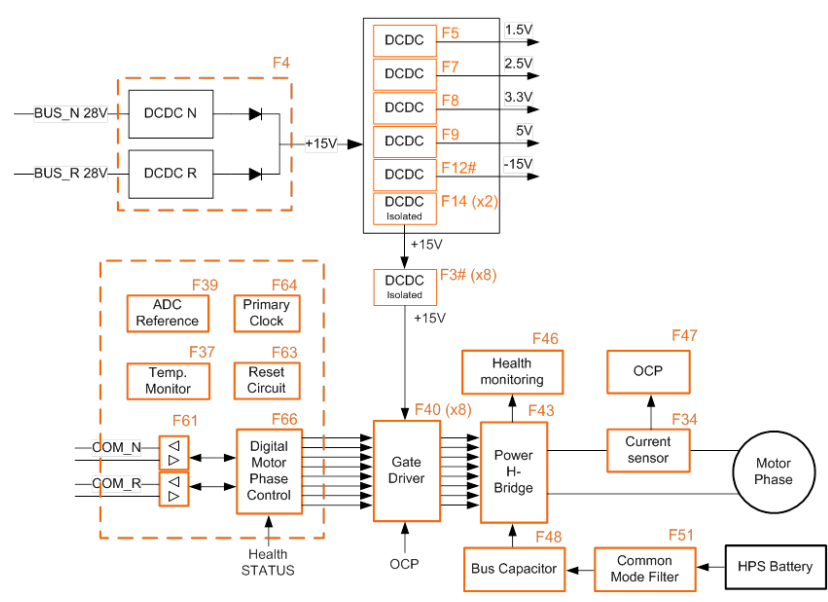

Figure 1. SPM functional architecture

Over-current protection is implemented in order to detect any malfunction into switching signals generation electronics. Both OCP detection and module switch-off are fully independent from the other functions.

A major concern before launcher take-off is to ensure that not any non-detectable failure has occurred during electronics integration. The result would be a start of mission with an electronics that is not single failure proof anymore and must be avoided. IGBT health monitoring has been therefore integrated.

Finally, a high capacity digital component is embedded in order to provide versatility when integrating the SPM into various applications.

\subsection{Fault tolerance}

A typical thrust vector control launcher application uses poly-phased motors, each phase driven by a SPM. The whole system being sized in such a way that loss of one phase keeps ensuring the full required performance.

The consequence is that loss of a SPM is allowed when facing a single component failure.

However, all SPM's being fed by a unique high power source (HPS), a single failure into a SPM may not cause any short-circuit on the HPS. This is especially 
applicable to power components composing the $\mathrm{H}$ Bridge where an IGBT failure could lead to mission loss.

A SPM has to be manageable by two nominal / redundant controllers and will thereby offer two separated control interfaces. There is no need for a single failure proof communication interface as communication links are made of a point to point network topology. In this case, loss of both communication channels leads to power module loss but has no impact on other modules used in parallel.

In order to avoid a counter-productive behaviour (e.g. a constant controlled current into a motor phase) of one module when losing control link, communication channel health has to be monitored and a time-out has to trigger power switch-off autonomously.

Finally, as a SPM is intended to be powered by two nominal/ redundant low power supplies, no single failure causing a short-circuit on both LPS is allowed as it would lead to loss of both N/R controllers.

\subsection{Main performance characteristics}

\section{- Electrical characteristics}

SPM average power output target is $5 \mathrm{~kW}$ and peak power is up to $10 \mathrm{~kW}$. It will thereby work at voltages up to $500 \mathrm{~V}$ and be able to deliver a peak current of $200 \mathrm{~A}$.

In order to provide the needed control bandwidth, its switching frequency will be up to $10 \mathrm{kHz}$, which is compliant with use of IGBT's.

\section{- Thermal Interfaces}

SPM design is foreseen to respect a temperature derating of $40^{\circ} \mathrm{C}$ wrt to maximum rating.

\section{Adiabatic mode:}

The module must be designed to work several minutes without transferring power to launcher structure.

It will thus make use of a high thermal capacity that will be sized to store dissipation caused by a delivery of 75 $\mathrm{A}_{\mathrm{RMS}}$ during 3 minutes with an initial temperature of $50^{\circ} \mathrm{C}$.

\section{Conductive mode:}

The SPM must also support longer missions, composed of high power delivery periods which will increase electronics temperature and quiet periods where low power is delivered allowing the temperature to decay.

SPM target is to limit thermal resistance between module baseplate and each power die to $0.5 \mathrm{~K} / \mathrm{W}$, a large analysis showing that this thermal resistance associated with the huge thermal capacitance will cover most of long mission needs.

\section{- Paschen effect}

Launcher applications present the specificity that powered-on electronic will see a depressurization from 1 bar to vacuum within a couple of minutes.

Working with voltages over $200 \mathrm{~V}$ into air implies to consider Paschen effect.

Thales Alenia Space has chosen not to put the Paschen constraint onto power modules but at equipment level by integrating all the electronics into airtight cases.

\section{- Environment}

Vibration and shock constraints are not presented here as they must be combined with equipment transfer function. Radiations and more particularly single event effect (SEE) have to be considered.

\section{SMART POWER MODULE DESIGN}

\subsection{Fault-tolerant power architecture}

A major concern of the power hybrid conception was the requirement forbidding power supply short-circuit in case of a single power component failure.

Protections using IGBT desaturation detection were ruled out as they have to be triggered within a dozen of microseconds and were thereby too sensitive to perturbations.

Moreover, use of desaturation detection based shortcircuit protection increases design power complexity as very high currents (up to several thousands of Amperes) have to be interrupted, causing higher overvoltages; this, in turn, demands active components with higher voltage ratings at the cost of switching and conduction losses increasing.

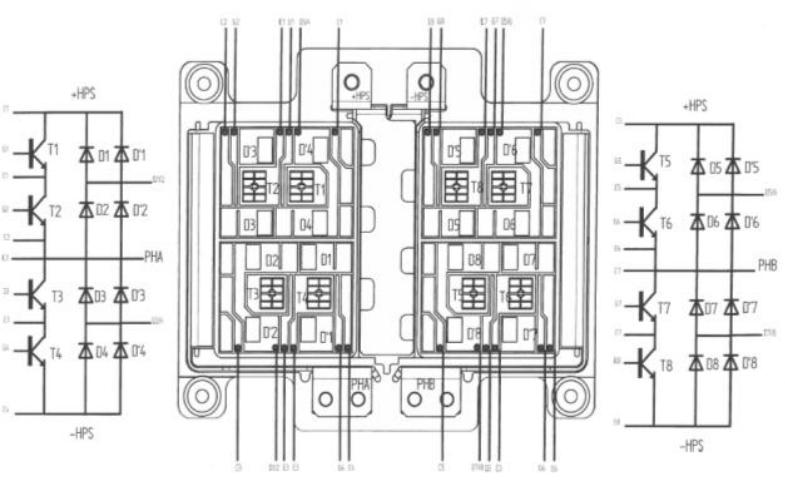

Figure 2 Fault tolerant power architecture

Considered alternative proposed in this design is the doubling of IGBT's in series (see Fig. 3). This solution has the advantage of preventing short-circuit instead of trying to isolate it, allowing the use of lower loss components.

Moreover, doubling the IGBT's in series increases robustness against SEE as both IGBT's of a same halfbranch must see a single event at the same time in order to provoke an effect.

\subsection{Physical segregation of power dies}

A dissipative power die failure may induce a very high and concentrated power emission and thus cause a high local temperature elevation. The impact could be failure propagation to other power dices. 
A first level of protection is provided by silicon gel which is poured within the hybrid.

A second level of protection against failure propagation is the use of separated substrates for each part of the Left/Right and Low/High half branches of the $\mathrm{H}$ bridge

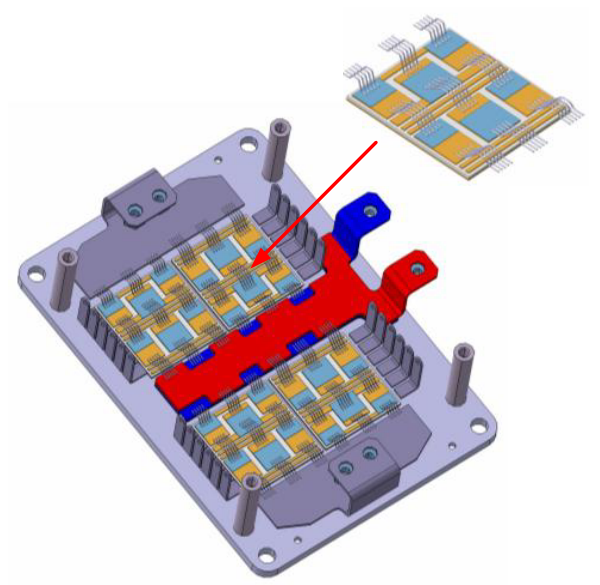

Figure 3: 4 identical substrates to compose H-bridge

However, laboratory experience has shown that these protections are not sufficient to border a high power default on a faulty die area.

As IGBT have been doubled in series, an additional physical segregation (walls) between each power dies of a substrate has been added.

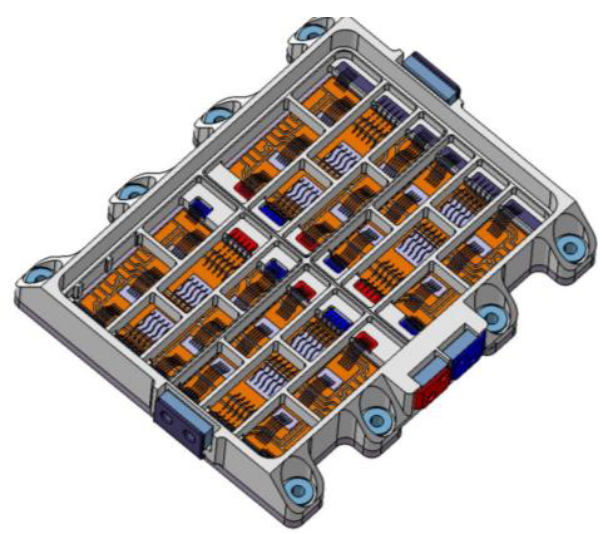

Figure 4: Physical segregation between dies

Thermal propagation in case of failure has been simulated and does not require any extra-protection.

\subsection{Optimized power dies implementation.}

When designing power hybrid substrates, a specific care has been taken to dies implementation.

An original way of implementing IGBT's and diodes dies to build-up a H-bridge, which lowers loop inductances, limits strongly overvoltages and EM emissions, especially at switching frequency, has been realized and patented by Thales Alenia Space.

The idea is to optimize currents paths and loops by placing diodes of the H-bridge lower side close to IGBT's of the higher side and vice-versa.

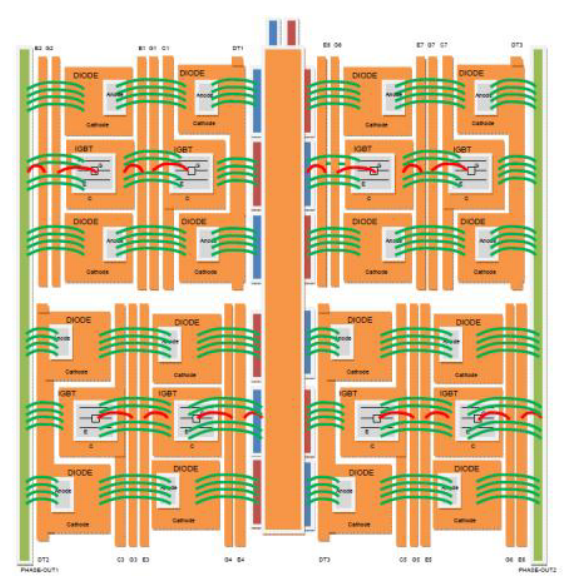

Figure 5: H-bridge diodes and IGBT dies patented implementation

A potential drawback is the vicinity of dies from different half bridge sides, leading to less segregation. However, this point has been mitigated by physical segregation of each die.

Tests results presented later show that this inventive topology reduces overvoltage in a very efficient way.

\subsection{COTS power module}

The first idea was the use of a COTS power module from industrial world. However, Fault-tolerance constraints did not allow the use of existing power modules.

The chosen alternative strategy was thus to design a custom hybrid, respecting the specific fault tolerance constraints and being producible by automotive large series production means, in order to keep cost under control.

\subsection{Use of automotive-proven technologies}

Fortunately, electrical and hybrid vehicles make a large use of power hybrids which are currently produced at a large scale while providing high-reliability and affordable costs.

The first phase of power design was the identification of power hybrid technologies already used into automotive industry and establish a design that could be produced by mature and highly automated production means.

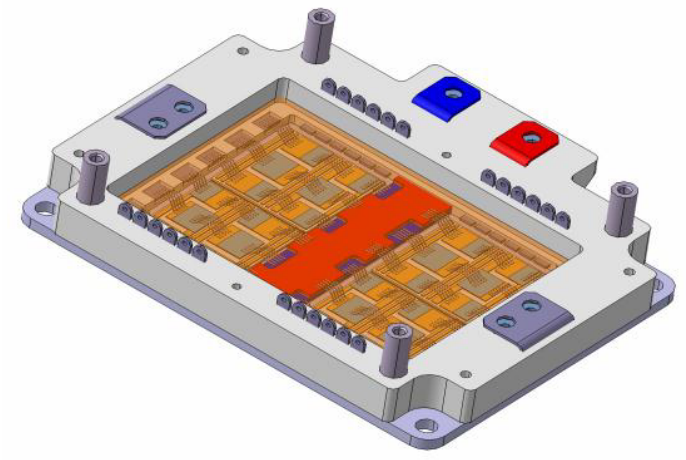

Figure 6: Power hybrid presentation 
The baseplate, made of copper, has been designed in such a way that it is able to store a high power dissipation during a typical launcher lower stage mission, that is, a few minutes.

Substrate technology is a DBC (Direct Bonded Copper) which is directly soldered on the copper baseplate. Dies are soldered on the DBC. Soldering of substrate and dies is aimed at optimizing thermal resistances.

A special focus has been set on the soldering process which has to limit voids under chip and substrate areas, voids increasing linearly thermal resistance path to the baseplate.

Interconnection of dies with substrates and substrate with package and bus-bars is carried out by means of an automated ultrasonic wire bonding process, using $\mathrm{Al}$ wires. Internal bus-bar lines are made of thick copper with aluminium cladding, central bus-bar being a superposed copper strip structure, required to be doubleisolated as it transfers energy from the unique HPS.

Housing is based on injected polymer resin around a copper structure in order to obtain an insulated package frame which will assure several functions:

- Protection of the electronic content from bad handling, or mechanical shocks, or vibration,...

- Good stiffness and stability

- Ability to be rigidly fixed to the copper base plate

Finally, an internal coating and protective gel has to be poured inside the device as to protect all internal conductors from being in contact with ambiance.

Different gels have been tested in order to ensure that they are compliant to vacuum environment and thermomechanical environment.

\subsection{Thermal aspects}

Before limiting thermal impact of dissipation on dice temperature by acting on thermal impedance, power dissipation has been limited as far as possible by using appropriate IGBT and diodes dies:

- $\mathrm{V}_{\mathrm{CE}}$ typ $<1.6 \mathrm{~V} @ 125^{\circ} \mathrm{C} / 150 \mathrm{~A}$

- E typ : $\mathrm{E}_{\mathrm{ON}}<1.75 \mathrm{~mJ}$

- $\mathrm{V}_{\mathrm{F}}<1.50 \mathrm{~V}$ typ @ $150 \mathrm{~A}, 125^{\circ} \mathrm{C}$

Detailed simulations of mission profiles show that worst case power dissipation of the whole power hybrid is more than $200 \mathrm{~W}$ in average and can reach up to $1 \mathrm{~kW}$ during a few milliseconds.

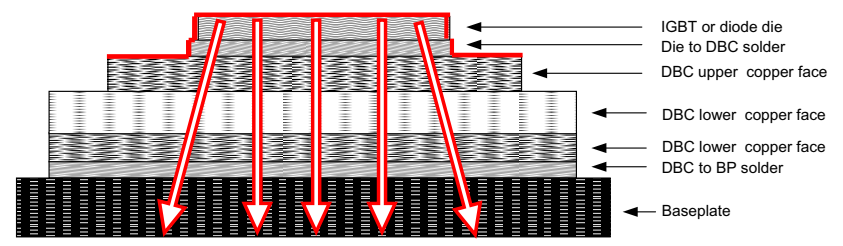

Figure 7: Thermal resistance between power die and baseplate is made of different layers.
At IGBT level, power dissipation is limited to around $40 \mathrm{~W}$ in average but could reach more than $300 \mathrm{~W}$ during a few milliseconds.

Conclusion of thermal analysis is that thermal design must achieve a thermal resistance of less than $0.5 \mathrm{~K} / \mathrm{W}$ per power component, from the die to the copper baseplate.

\subsection{Power hybrid integration with SPM structure}

A SPM has to be produced in large series (up to 1000 pieces per year) and has therefore to be simple to assemble.

The number of elements and the number of different articles has been kept as low as possible in order to be able to produce it in the simplest way.

Power drive electronics being partially at high power potential, they have been put on a dedicated PCB in order not to interfere and limit high voltage design constraints on low level electronics.

The result is an easy to produce compact module, which is simple to integrate into more complex applications.

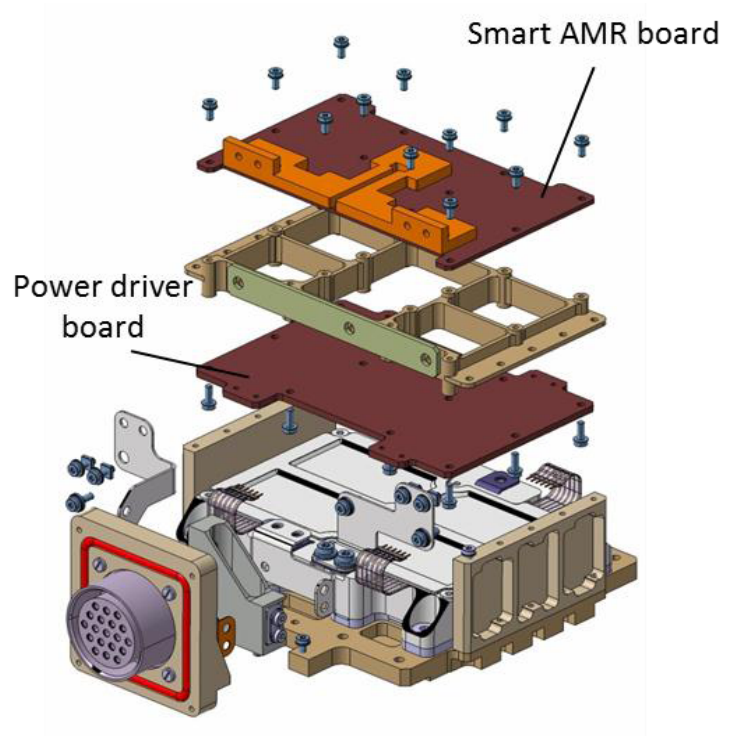

Figure 8: SPM mechanics

\section{TESTS}

\subsection{Test goals}

The test campaign pursued the following objectives:

- To demonstrate that a modular concept is adapted to multi-phases motor drive applications.

- To measure overvoltage spikes due to electronics switching and confirm thereby voltage margins.

- To confirm thermal studies estimating the worst case end of mission power dies temperature around $110^{\circ} \mathrm{C}$ for a launcher low stage typical mission. 


\subsection{Mock-up}

An electrical and thermal representative mock-up, whose base element is a high-reliability automotive quality power hybrid prototype, has been built-up, made of six SPM's and driven through their communication bus by one FPGA based controller.

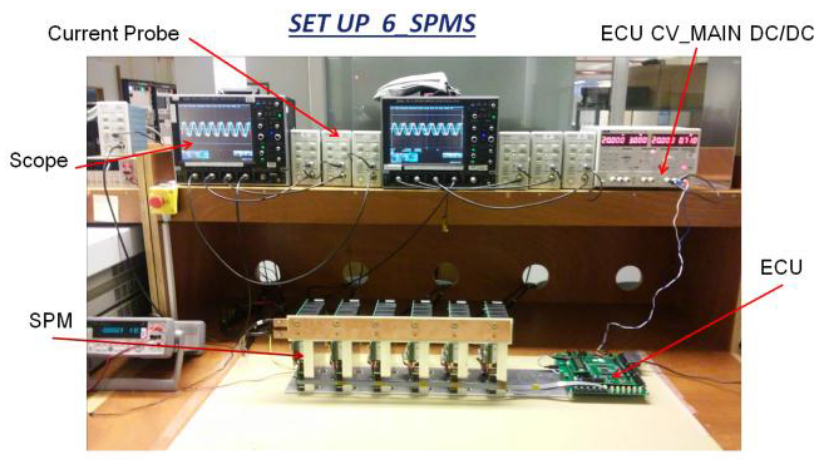

Figure 9: SPM prototype integration into motor drive application.

The controller sends appropriate duty cycles to SPM's in order to generate 6 sinusoidal currents in each of six air-inductances, each current being shifted by $60^{\circ}$.

This test is representative of a six-phased synchronous motor drive application, which is typical of a $60 \mathrm{~kW}$ launcher electromechanical actuator application.

\subsection{Tests results}

\section{- Multiphase motor application:}

Conclusion is that both communication link bandwidth and embedded electronics allow to generate currents with amplitudes and bandwidth which are compatible to targeted applications.

\section{- Switching overvoltage:}

Overvoltage generated is strongly limited to a few dozens of Volts which is a very interesting improvement factor with regards to common IGBT power modules.

\section{- End of mission temperature:}

Typical lower stage launcher RMS Current levels have been injected for a period of 3 minutes, temperatures are in line with expected results :

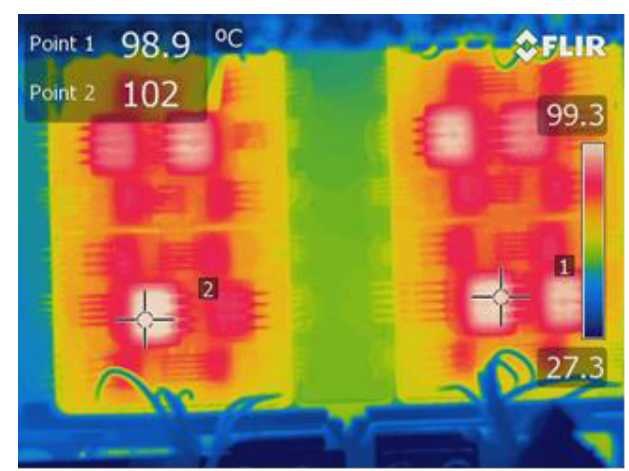

Figure 10: Power dies temperature at end of mission.

\section{CONCLUSIONS}

This paper has presented the design of a multipurpose smart power module intended to answer to a large number of needs into launcher applications.

Specific launcher RAMS constraints have been considered and the way the module fits these requirements demonstrated.

Technologies from automotive industry have been used as far as possible in order to reduce production costs and have been detailed .

Remarkable fact are that overvoltage level related to power switching is kept to a very low level, thanks to an innovative dies implementation and that thermal performances are very good, comparable to COTS power module.

This automotive quality level concept could be extended to satellite application into a next future.

\section{REFERENCES}

[1]. C. Lihua, F. Z. Peng, and C. Dong (2008), “A smart gate drive with self-diagnosis for power MOSFETs and IGBTs", in Proc. 23rd Annu. IEEE Appl. Power Electron. Conf. Expo., pp. 1602-1607.

[2]. V. Choudhary, E. Ledezma, R. Ayyanar, and R. M. Button (2008), "Fault tolerant circuit topology and control method for input-series and outputparallel modular DC-DC converters", IEEE Trans. Power Electron., vol. 23, no. 1,pp. 402-411.

[3]. B. Francois and J. P. Hautier (2002), "Design of a fault tolerant control system for a N.P.C. multilevel inverter", in Proc. IEEE Int. Symp. Ind. Electron. L'Aquila, Italy, vol. 4, pp. 1075-1080.

[4]. B.A. Welchko, TA. Lipo, ,T.M Jahns, S.E. Schulz (2004) : "Fault tolerant three-phase AC motor drive topologies, a comparison of features, cost and limitations", IEEE Transactions on Power Electronics, Vol.19, Issue4, pp.1108-1116. 